\title{
The Effect of Coffee and Tea on Blood Pressure of University Students
}

\author{
Uyanga Sumiya \\ Bentley University. 175 Forest St. \\ Waltham, MA 02452 U.S.A. \\ Hanyin Ni \\ Bentley University. 175 Forest St. \\ Waltham, MA 02452 U.S.A. \\ Paul D. Berger \\ Bentley University. 175 Forest St. \\ Waltham, MA 02452 U.S.A.
}

\begin{abstract}
We collected data on blood pressure from randomly selected university students before and after they consumed a caffeinated beverage. The experiment was a two-level two-factor complete-factorial design with eight replicates. One factor is gender with Female and Male as the levels. The other factor is drink type with Coffee and Black Tea as the levels. There was no effect on the systolic blood pressure (SBP) and the diastolic blood pressure (DBP) due to the different caffeine drinks (coffee or black tea). Gender was a significant factor that affects the SBP and the DBP. The interaction effect of caffeinated drink and gender was not significant for either blood pressure measurement.
\end{abstract}

\section{INTRODUCTION}

On-campus cafes are popular for university students who purchase coffee, tea and other beverages every day. Coffee and tea are the main caffeine-composed drinks. Many students take caffeinated drinks to increase their energy and alertness; however, caffeine may have negative effects on health. The common understanding of the effect of caffeine is to elevate blood pressure. Some experiments found a positive relation between caffeine and cardiometabolic risk factors (Abrahao et al., 2013). However, most research exhibits mixed results of the effect of coffee and tea consumption on human health. Thus, we conducted an experiment for whether drinking coffee or tea has a different effect on university students' blood pressure.

\section{LITERATURE REVIEW}

Coffee is one of the most popular beverages consumed in the world. This is especially true in the U.S. It is consumed regularly on a daily basis. Sixty-two percent of adults in America consume on average three cups of coffee per day (Kumar \& Verma 2013). A cup of coffee can contain 100-150 mg of caffeine. Caffeine induces various acute cardiovascular effects, such as an up-regulation of circulating catecholamines, leading to increases in systolic (SBP) and diastolic (DBP) blood pressure (Kumar \& Verma 2013). Caffeine enters all tissue compartments, and through its actions at the adenosine receptor, it has widespread effects on the central nervous system and all peripheral tissues. Ultimately, it triggers sudden energy and alertness. Although caffeine is a primary compound of coffee, there are several other 
biologically active components that may or may not be harmful to a cardiovascular system. Of course, decaffeinated coffee is often available - although not part of our study.

One of the potential substitutes to coffee is tea. Tea contains a lower amount of caffeine, and different compounds, compared to coffee, and tea is considered to have many health benefits. A cup of regular tea can contain 14-61 mg of caffeine depending on the steeping time. Black tea contains the highest amount of caffeine - about 59-61 mg per cup. Black tea is made from the leaves of the plant, Camellia Sinesis, and is generally stronger in flavor and more oxidized than green tea (Walli et al., 2015). The result of few studies investigating the relationship between regular tea consumption and blood pressure have been inconsistent. A study conducted in Norway found that higher consumption of black tea was associated with lower systolic blood pressure (Stensvold et al., 1992). However, it was suggested that on top of caffeine in tea, oxidative stress can be involved in the development of increased blood pressure (RomeroAlvira \& Roche, 1996).

Blood pressure may differ depending on gender. Alhawari et al. (2018) found significant differences in both SBP and DBP between male and female participants. Males tended to have higher blood pressure compared to females. Moreover, more men suffer from high blood pressure (hypertension) than women. A study conducted by Everett \& Zajacova (2015) among men and women in their twenties found that women were less likely to be hypertensive compared to men. Therefore, an aim of our study is to determine whether there is a gender difference in blood pressure when drinking coffee vs. tea.

\section{Subjects and experimental design}

\section{METHODOLOGY}

We recruited thirty-two university students currently studying at two universities in Massachusetts, United States. Participants were selected randomly. The students aged between 19 and 38. Each beverage was consumed by 8 male and 8 female students, yielding 32 data points. Students were advised not to drink any caffeine-containing beverages for at least 6 hours before attending the experiment. Heart rate, and systolic and diastolic blood pressures, were measured before they began each drink, as well as 20 minutes after they completed the drink. The experiment was a two-level two-factor complete factorial design, with eight replicates per treatment combination. The experiment took place in November, 2018.

\section{Preparation of the beverages}

Black tea (Tazo Awake) and black coffee (Starbucks Caffe Americano) were selected as the experiment materials. Six ounces of the chosen black tea steeped for 1 minute contains $59 \mathrm{mg}$ of caffeine. The same amount of the selected coffee contains $75 \mathrm{mg}$ of caffeine. Both beverages were served hot, with average temperature between 96-104F (35-40C).

\section{Blood pressure measurement}

Blood pressures were measured using a CareTouch fully automated blood pressure monitor. The blood pressure and heart rate were recorded on the left arm with the subjects in a sitting position. The subjects stayed calm and relaxed. Most of the subjects finished their drink within 5 minutes.

\section{Statistical analysis}

The dependent variable is the blood pressure measurements. The two factors under study are gender and caffeinated drink type. Each factor has two levels. "Gender" factor has "Male" and "Female" as its levels, and "Drink type" factor has "Coffee" and "Tea" as its levels. 
All statistical analyses were performed using R software. Two-way ANOVA was used to analyze the mean difference of systolic and the mean difference of diastolic blood pressure between different gender groups and different caffeine drink types. Type I error of .05 (i.e., $\alpha=.05$ ) was used for the statistical test. A p-value less than 0.05 was considered statistically significant, meaning that the mean is different between the two cohorts being compared.

\section{RESULTS}

Table 1 provides a summary of the data results.

Table 1: Mean of each cell for three different measures*

\begin{tabular}{llll|ll|l}
\hline \multirow{2}{*}{} & & \multicolumn{2}{c|}{ Coffee } & \multicolumn{2}{c|}{ Tea } & \multirow{2}{*}{ Overall } \\
& & Female & Male & Female & Male & \\
\hline \multirow{3}{*}{ Before } & Heart rate & 77.8 & 75.5 & 79 & 76.5 & 77.2 \\
& Systolic (mmHg) & 123 & 141 & 116 & 131 & 127.7 \\
& Diastolic (mmHg) & 76.8 & 93.5 & 72.9 & 85.5 & 82.2 \\
\hline \multirow{3}{*}{ After } & Heart rate & 79.2 & 73.2 & 76.6 & 74.2 & 75.8 \\
& Systolic (mmHg) & 121 & 144 & 122 & 136 & 130.8 \\
& Diastolic (mmHg) & 77.1 & 94.9 & 78.6 & 86 & 84.2 \\
\hline
\end{tabular}

* Values for coffee and tea to nearest integer for Systolic, to tenths digit for other two measures.

According to Table 1, the overall average SBP increased from $127.7 \mathrm{mmHg}$ to $130.8 \mathrm{mmHg}$ after drinking the beverage, whereas, the average DBP increased from $82.2 \mathrm{mmHg}$ to 84.2 $\mathrm{mmHg}$. For female participants who consumed coffee, the average SBP decreased by $2 \mathrm{mmHg}$, and the average DBP increased by $0.3 \mathrm{mmHg}$. The average SBP, and the average DBP, of male coffee drinkers increased by $3 \mathrm{mmHg}$ and $1.4 \mathrm{mmHg}$, respectively. As opposed to the coffee drinkers, the average SBP and the average DBP of tea drinkers increased among both male and female participants. The average SBP and the average DBP increased by $6 \mathrm{mmHg}$ and 4.7 $\mathrm{mmHg}$, respectively for females who consumed tea, whereas, they increased by $5 \mathrm{mmHg}$ and $0.5 \mathrm{mmHg}$ respectively for male tea consumers. Although the descriptive analysis exhibited an increase in the blood pressure for both beverages, further statistical analysis was required to make conclusions about the statistical significance of the findings. Table 2 presents these results.

Table 2. F-statistic Results of Two-way ANOVA

\begin{tabular}{lcc}
\hline & $\begin{array}{c}\text { Systolic blood } \\
\text { pressure }\end{array}$ & $\begin{array}{c}\text { Diastolic blood } \\
\text { pressure }\end{array}$ \\
\hline Drink type & 0.219 & 0.521 \\
Gender & $5.639^{* *}$ & $6.045^{* *}$ \\
$\begin{array}{l}\text { Interaction of Drink and } \\
\text { Gender }\end{array}$ & 0.284 & 1.031
\end{tabular}

* Sig at $10 \%,{ }^{* *}$ Sig at $5 \%,{ }^{* * *}$ Sig at $1 \%$

A two-way ANOVA test was conducted to test the effect of the "Drink type" factor (coffee and tea) and the "Gender" factor (male and female) on blood pressure. The measurements of blood pressure after the consumption of the caffeine beverages were used as the response/dependent variable to be tested. According to Table 2, "Drink type" was not 
significant for SBP and DBP at the 5\% significance level. On the contrary, "Gender" was statistically significant at the 5\% significance level. The interaction of "Drink type" and "Gender" was not significant for both SBP and DBP at the 5\% significance level. The test results indicated that the blood pressure after the consumption of coffee vs. tea did not differ significantly, although the absolute value increased. "Gender," however, had a significant effect on both blood pressure measures, which suggests that there's a significant difference in blood pressure between males and females. There is no indication of an interaction effect between "Gender" and "Drink type."

Next, and perhaps more meaningful, the change in blood pressure was analyzed. The difference between the after and before blood pressures was used as the dependent variable. Table 3 displays the differences and the significance of the results. Perhaps we should say "non-significance," as none of the effects were significant at $\alpha=.05$.

Table 3: Mean difference and significance of results

\begin{tabular}{lcc|cc}
\hline & $\begin{array}{c}\text { Systolic blood } \\
\text { pressure } \\
\text { Coffee }\end{array}$ & Tea & Coffee & Tea \\
\hline Male & 3 & 5 & 1.4 & .5 \\
Female & -2 & 6 & 3 & 5.7 \\
\hline
\end{tabular}

* Sig at $10 \%,{ }^{* *}$ Sig at $5 \%,{ }^{* * *}$ Sig at $1 \%$

In Table 3, the male group who consumed coffee had increased SBP and DBP by $3 \mathrm{mmHg}$ and $1.4 \mathrm{mmHg}$, respectively. However, the changes were not statistically significant at $5 \%$ significance level. The male tea consumers had their SBP and DBP increased by $5 \mathrm{mmHg}$ and .5 $\mathrm{mmHg}$, respectively; however, these values were also not significant at $5 \%$ significance level. As for the female cohorts, the coffee drinkers' SBP dropped by $2 \mathrm{mmHg}$ and their DBP increased by $3 \mathrm{mmHg}$, respectively. The female tea drinkers' SBP and DBP were raised by 6 $\mathrm{mmHg}$ and $5.7 \mathrm{mmHg}$, respectively; none of these changes were significant.

\section{DISCUSSION}

The study found that blood pressure significantly differs between males and females, which confirms the result of previous research. In terms of the change in blood pressure associated with either coffee or tea, there's no evidence to conclude that the effect of one caffeinated drink type is different than the other type; in fact, neither drink showed a significant effect. It could be the development of tolerance to caffeine during habitual use that may explain the reason why the consumption of coffee or tea did not affect the blood pressure of the participants. Also, the timing of intake over the day could influence the overall effect of caffeine on blood pressure. It is possible that the brewing method contributed to a different amount of caffeine in the drink types, and added to the variance of the results, leading to a potential masking of significant results.

The insignificant results of for change in blood pressure after the consumption of the beverages suggest that the habit of drinking coffee and tea may cause physical or mental relaxation or, conversely, abstinence may induce a level of discomfort, which could influence blood pressure (Noordzij et al., 2005). Inter-individual variability in the rate of absorption and effect of caffeine might be relevant factors. 


\section{LIMITATIONS AND DIRECTIONS TO FUTURE RESEARCH}

Our conclusions are based on the data we collected among the students in the Northeast of the U.S. It is possible that there is a location bias. In addition, more demographic, life style, or dining preference as additional factors, might enhance the analysis, but they were not provided by the subjects due to privacy concerns.

However, the major limitation in our analysis may be that the difference in caffeine content was not that different between the two drinks. An average $59 \mathrm{mg}$ for the tea, and $71 \mathrm{mg}$ for the coffee, is not that big a difference. Also, the absolute amounts were not that high. If the experiment were to be repeated, it would be useful to the granularity of the study to increase both the absolute amounts of the caffeine, and perhaps more important, the difference between the amount of caffeine in the two drinks - even if some of the difference is arrived at by having different amounts of ounces of liquid in the two treatments. Overlooking that two levels are too close to one another to find a significant difference is not that uncommon an "oversight." There have been many examples of such an occurrence - especially in pricing experiments, in which the two "levels" of price are too close together to make a difference to the consumer in his/her decision making, and other factors, such as warranty or appearance, dominate.

For future studies, we would also suggest that one explores the caffeine effect on university students between different racial groups, and the study takes exercise routines, diets and family history, etc., into account as covariates. Finally, the experiment was conducted in the winter of 2018, when the weather was cold in the Northeast of the U.S., and when university students were beginning to get busy preparing for the exams. It might be preferable to conduct the experiment in different seasons to exclude the possible weather factor and emotional factor.

Another aspect that we observed in our study was that 11 students (34\%) were potentially hypertensive - i.e., stage 1 hypertension. A participant was considered hypertensive if his/her blood pressure was outside of the diagnostic range of 130/80 $\mathrm{mmHg}$ according to the guideline of the American Heart Association and the American College of Cardiology. Future research may wish to consider hypertension in the study.

\section{References}

Abrahao. S. A, Pereira. R. G, de Sousa. R. V, Lima. A. R, Crema. G. P \& Barros. B. S. (2013). Influence of coffee brew in metabolic syndrome and type 2 diabetes. Plant Foods for Human Nutrition, 68, p. 184-189.

Alhawari. H. H., Al-Shelleh. S., Alhawari. H. H., Al-Saudi. A., Al-Majali. D. A., Al-Faris. L. \& Al-Rvalat. S. A. (2018). Blood pressure and Its Association with Gender, Body Mass Index, Smoking, and Family History among University Students. International Journal of Hypertension.

Americans Are Drinking More Coffee Than Ever. (n.d.). Retrieved from https://www.foodandwine.com/news/americans-drinking-more-coffee-ever

Black Tea. (n.d.). Retrieved from https://www.caffeineinformer.com/caffeine-content/tea-brewed

Everett. B. \& Zajacova. A. (2015). Gender Differences in Hypertension and Hypertension Awareness Among Young Adults, Biodemography Soc Biol., 61, p 1-17.

Hodgson. J. M., Puddey. I. B., Burke. V, Beilin. L. J. \& Jordan. N (1999). Effects on blood pressure of drinking green and black tea. Journal of Hypertension, 17, p. 457-463.

Jiang. X, Zhang. D \& Jiang. W. (2014). Coffee and caffeine intake and incidence of type 2 diabetes mellitus: a metaanalysis of prospective studies, European Journal of Nutrition, 53, p. 25-38.

Kumar. P. \& Verma. D. K. (2013) Effect of coffee on blood pressure and electrocardiographic changes in young and elderly healthy subjects. National Journal of Medical Research, 3(1),p. 53-55. 
Noordzij. M., Uiterwaal. C., Arends. L. R., Kok. F. J., Grobbee. D. E. \& Geleijnse. J. M. (2005). Journal of Hypertension, 2, p. 921-928.

Romero-Alvira D, Roche E. (1996), High blood pressure, oxygen radicals and antioxidants: etiological relationships. Med Hypoth, 46:414 \pm 420 .

Stensvold I, Terdal A, Solvol K, Foss OP. Tea consumption. Relationship to cholesterol, blood pressure and coronary and total mortality (1992), 21:546 \pm 553 .

The Complete Guide to Starbucks Caffeine. (n.d.). Retrieved from https://www.caffeineinformer.com/thecomplete-guide-to-starbucks-caffeine

Walli. R. R., Almosrati. R. A., Zaied. A. A., Shummakhi. F, Bredae. E. G. \& Shalaka. O. K. (2015). The Relationship between Habitual Coffee and Tea Consumption and Type 2 Diabetes Mellitus among Libyan Adults. International Journal of Pharma Research \& Review, 4(5), p. 34-39. 